\title{
Link Budget Analysis of In-body to On-body UWB Low Band Communications for Capsule Endoscope
}

\author{
Jingjing Shi \\ Graduate School of Engineering \\ Nagoya Institute of Technology \\ Nagoya 466-8555 \\ Japan \\ chx17507@stn.nitech.ac.jp
}

\author{
Daisuke Anzai \\ Graduate School of Engineering \\ Nagoya Institute of Technology \\ Nagoya 466-8555 \\ Japan \\ anzai@nitech.ac.jp
}

\author{
Jianqing Wang \\ Graduate School of Engineering \\ Nagoya Institute of Technology \\ Nagoya 466-8555 \\ Japan \\ wang@nitech.ac.jp
}

\begin{abstract}
This paper aims to perform a link budget analysis for a typical application in wireless implant body area networks (BANs), i.e., capsule endoscope. Ultra wideband (UWB) technology is an attractive candidate for wireless body area communications, especially for providing a high data rate to fulfill real time transmission. In this study, based on the channel characterization and average bit error rate (BER) evaluation results in pulse position modulation (PPM) or on-off keying modulation impulse radio (IR) UWB system over in-body to on-body communication channel, system margin on both single fading channel and twobranch diversity channel are firstly derived and compared, then the link parameters including the communication distance, data rate as well as required transmit power are discussed to clarify the link budget improvement with adoption of diversity reception technique.
\end{abstract}

\section{Categories and Subject Descriptors}

C.2.1 [Computer-Communication Networks]: Network

Architecture and Design-Wireless communication

\section{General Terms}

Performance

\section{Keywords}

Link budget, in-body to on-body communication, ultra wideband (UWB), spatial diversity, bit error rate (BER).

\section{INTRODUCTION}

Ultra wideband (UWB) is a technology which has been drawn considerable consideration recently in a wide range of applications especially for body area networks (BANs) where devices locate on or in the proximity of a human body [1]. For a very typical medical application such as capsule endoscope in implant BAN, a reliable communication link and a high data rate up to $10 \mathrm{Mbps}$ are in urgent requirement to realize real time image observations [2]. The use of UWB technology can offer these possibilities and become a strong candidate in the wireless standard on BAN. Based on our recent research results for UWB in-body propagation [3] [4], however, it is understood that inbody transmission has to be limited in the UWB low band, for example from 3.4 to $4.8 \mathrm{GHz}$ as defined in Japan. That is because with the increasing radio frequency, skin depth or penetration depth of human tissue becomes very small. Different from wearable BAN communication, implant BAN mainly undergoes severe signal decay during transmission which may lead to undesired communication performance degradation. In order to improve the quality and reliability of UWB in-body communication, a spatial maximal ration combining (MRC) diversity reception technique has been adopted [5].

The objective of this study is to conduct a link budget analysis for PPM-IR-UWB and OOK-IR-UWB wireless communication system of capsule endoscope application. At first, we derive system safety margins versus communication distance in both single in-body fading channel and two-branch in-body MRC diversity channel based on our previous study results on channel characterization and BER performance evaluation, then we discuss the communication parameters such as communication distance, data rate as well as required transmit power over in-body communication channels. Finally we clarify the effective improvement of link budget with diversity technique. This work contributes to the understanding of the feasibility of capsule endoscope in UWB low band and practical system design.

\section{LINK BUDGET ANALYSIS MODEL}

Figure 1 shows an anatomically based human body model with an on-body elliptic disc dipole antenna to receive the capsule endoscope data. The body model was developed based on magnetic resonance imaging data, and has nearly 50 types of tissue with a spatial resolution of 2-mm [6]. The receiving antenna was assumed at five locations in the front of abdomen for spatial diversity reception, i.e., $\mathrm{Rx} 1 \& \mathrm{R} \times 3$, $\mathrm{Rx} 1 \& \mathrm{Rx} 5$ as shown in the figure. On the other hand, the transmitting antenna of the capsule endoscope was assumed as a 4-mm long dipole. It was moved along the small intestine at 33 locations, and at each location it had three different directions ( $\mathrm{x}, \mathrm{y}$, and $\mathrm{z}$ ) respectively. For each on-body receiver location, the path loss and shadow fading characteristics were derived from 99 data using the finite difference time domain (FDTD) method in conjunction with the anatomical human body model [7]. Then a two-path impulse response model was proposed and it made us easy to adopt a noncoherent envelop or energy detection at the receiver. BER 
performance evaluation has also been clarified in [5]. The link budget [8] in this case is the accounting of all of the gains and losses from the transmitting dipole antenna of capsule endoscope, through the in-body fading channel to the receiving antenna in this case as shown in Figure 1.
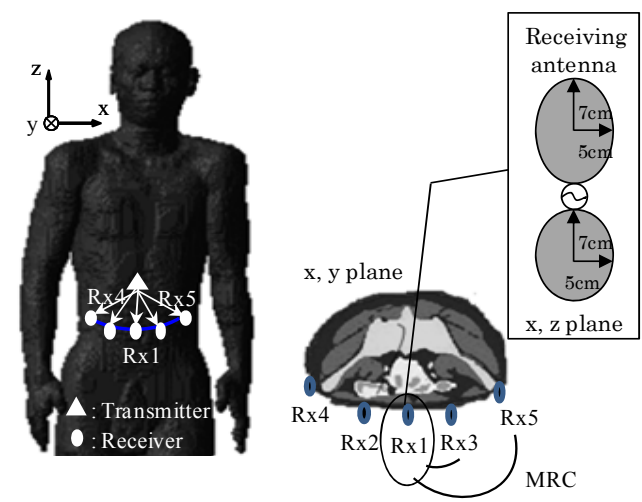

Figure 1. Link budget analysis model with diversity reception.

\section{SYSTEM MARGIN}

In order to analyze the link budget for in-body UWB channel, besides the derived path loss, the noise characteristics and the possible transmitted signal power also have to be calculated. The noise situation in a receiver depends on several noise sources. To simply this situation, we assume a single equivalent noise source as additive white-Gaussian-noise (AWGN). This noise is typically thermal, introduced by the receiving antenna and the front-end circuit of the receiver. The thermal noise power spectrum density (PSD) expressed in $\mathrm{W} / \mathrm{Hz}$ is given by:

$$
N_{0}=k T_{a}+k\left(N_{F}-1\right) T_{0}
$$

where $T_{a}$ is the receiving antenna temperature; $T_{0}=300 \mathrm{~K}$ is the environment temperature; $k=1.38 \times 10^{-23} \mathrm{~J} / \mathrm{K}$ is the Boltzmann constant; and finally, $N_{F}$ is the noise figure of the receiving device (assumed as $6 \mathrm{~dB}$ ). Since the receiving device is adorned on the human body, a reasonable hypothesis is introduced as $T_{a}=T_{0}=300 \mathrm{~K}$. Equation (1) can therefore be rewritten in decibel as

$$
N_{0, d B}=10 \log _{10}\left(k T_{0}\right)+N_{F, d B}=-198[\mathrm{dBW} / \mathrm{Hz}]
$$

Since the FCC limits the emission power to be $-41.3 \mathrm{dBm} / \mathrm{MHz}$ in the allowable band for UWB systems, the maximum allowed transmit power should be $0.1 \mathrm{~mW}$ or $-10 \mathrm{dBm}$ for the in-body to on-body communication at UWB low band (1.4 GHz bandwidth). The FCC regulation illustrates the fact that the UWB signal must meet the emission mask and the maximum allowed transmit power requirements. Signal power up to the maximum allowed transmit power can be considered as the best case in performance analysis for the UWB system. Given the allowed maximum power, we can evaluate system margin when a predetermined probability of error must be guaranteed at the receiver.

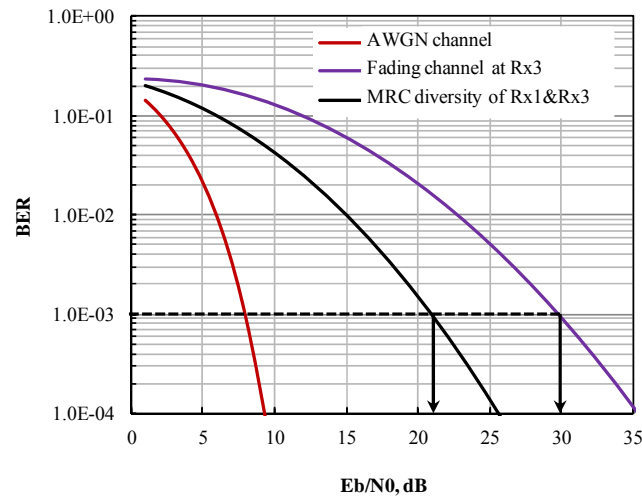

(a) PPM scheme

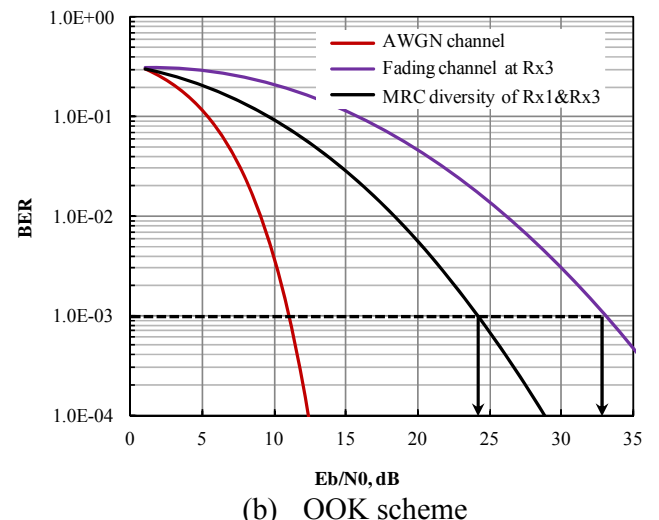

Figure 2. Average BER performances for IR-UWB system with PPM and OOK schemes and non-coherent detections.

On the other hand, the received power at each receiver location can be expressed using the path loss model without considering shadow fading as

$$
\begin{aligned}
P_{r, d B W} & =P_{t, d B W}-P L_{d B} \\
& =P L_{0, d B}-10 n \log _{10}\left(d / d_{0}\right)
\end{aligned}
$$

where $P L_{0, d B}$ is the reference path loss $(48.5 \mathrm{~dB})$ at the distance $d_{0}=5 \mathrm{~cm}, n$ is the path loss exponent and was introduced as 8 at $\mathrm{Rx} 3$, and $d$ indicates communication distance. It should be noted that in equation (3), transmitting and receiving antenna gains have been considered when deriving the path loss model. Under the given modulation technique of PPM or OOK scheme, the link signal-to-noise power ratio (SNR) or $E_{b} / N_{0}$ which is necessary for a receiver to achieve a specified level of reliability in terms of BER in decibel can be derived as

$$
\left[E_{b} / N_{0}\right]_{d B}=P_{r, d B W}-10 \log _{10} f_{b}-N_{0, d B}
$$

where $f_{b}$ is the frequency bandwidth or data rate. Thus, we can define the system margin for this in-body to on-body UWB channel as $M_{s}$ by

$$
M_{s}=\frac{E_{b} / N_{0}}{\left[E_{b} / N_{0}\right]_{\text {spec }}}
$$

where $\left[E_{b} / N_{0}\right]_{\text {spec }}$ denotes the required $E_{b} / N_{0}$ for obtaining a specific probability of error. Here we use BER $=0.001$ as the 
predetermined probability of error threshold according to error correction theory [9]. As shown in Figure 2, we can get the required $E_{b} / N_{0}$ of IR-UWB with PPM and OOK schemes for non-coherent detection with $\mathrm{BER}=0.001$. The corresponding values are $30 \mathrm{~dB}$ and $33 \mathrm{~dB}$, respectively, without diversity for single fading channel at $\mathrm{Rx} 3$, and $21 \mathrm{~dB}$ and $24 \mathrm{~dB}$, respectively, with MRC diversity of Rx1 and Rx3. In equation (5), if the link $E_{b} / N_{0}$ exceeds the required $\left[E_{b} / N_{0}\right]_{\text {spec }}$, which means system margin $M_{s} \geq 0 d B$, the wireless communication is feasible to be realized. The larger the system margin, the more reliable communication system. Since with the maximum allowed transmit power the best case can be expected in performance analysis for the UWB system, we assumed $P_{t, d b m}=-10 d B m$ in the derivation process of system margin. The parameters for in-body to on-body UWB link budget analysis are summarized in Table 1 .

Table 1. Parameters for in-body to on-body UWB link budget analysis

\begin{tabular}{|c|c|c|}
\hline \multicolumn{3}{|c|}{ Transmitter \& Receiver } \\
\hline \multicolumn{2}{|c|}{ Frequency [GHz] } & $3.4-4.8$ \\
\hline \multicolumn{2}{|c|}{ Transmitter output power $P_{t}[\mathrm{dBm}]$} & -10 \\
\hline \multicolumn{2}{|c|}{ Standard temperature $T[\mathrm{~K}]$} & 300 \\
\hline \multicolumn{2}{|c|}{ Receiver noise figure $N_{F}[\mathrm{~dB}]$} & 6 \\
\hline \multicolumn{2}{|c|}{ Boltzmann constant $[\mathrm{J} / \mathrm{K}]$} & $1.38 \mathrm{E}-23$ \\
\hline \multicolumn{3}{|c|}{ Signal quality } \\
\hline Bit error rate & \multicolumn{2}{|l|}{0.001} \\
\hline$E_{b} / N_{0} \quad[\mathrm{~dB}]$ & \multicolumn{2}{|c|}{$\begin{array}{l}30 \text { (PPM, non-coherent detection ) } \\
33 \text { (OOK, non-coherent detection ) } \\
21 \text { (PPM, MRC diversity of Rx1\&Rx3) } \\
24 \text { (OOK, MRC diversity of Rx1\&Rx3) }\end{array}$} \\
\hline
\end{tabular}

Under the assumption of system parameters tabulated in Table1 and the above equations, we can derive the system margin versus communication distance at different data rate. Figures 2 and 3 show the dependence of system margin on link distance with data rates of $0.1 \mathrm{Mbps}, 1 \mathrm{Mbps}$ and $10 \mathrm{Mbps}$ for IR-UWB with PPM and OOK, respectively. It can be found that, at a data rate of 1 Mbps, the system has a margin of at least above $0 \mathrm{~dB}$ for PPM at a distance of $8.8 \mathrm{~cm}$ and for $\mathrm{OOK}$ at a distance of $8 \mathrm{~cm}$ both from the body surface. If the data rate is increased to $10 \mathrm{Mbps}$, the possible communication distance will be limited to only $6.6 \mathrm{~cm}$ for PPM and $6 \mathrm{~cm}$ for OOK. These results suggest that a further BER improvement is necessary to make the data transmission from the digestive organs possible. Figures 4 and 5 show the dependence of system margin on link distance with the MRC diversity reception of $\mathrm{Rx} 1 \& \mathrm{R} \times 3$. Such an improved receiver structure provides a better system margin. As can be seen, in almost all of the transceiver locations inside the digestive organs which are usually within $15 \mathrm{~cm}$ from the body surface, a data rate of 0.1 Mbps can always have a system margin above $0 \mathrm{~dB}$ for PPM with 2-branch MRC diversity. When the data rate is increased to $1 \mathrm{Mbps}$ and $10 \mathrm{Mbps}$, however, the corresponding communication distances will be reduced to $11.4 \mathrm{~cm}$ and $8.6 \mathrm{~cm}$, respectively. The possible communication distance for OOK scheme is somewhat shorter compared to PPM scheme? The maximum link distances at different data rates considered in this study are summarized in Table 2 . It has been confirmed that the use of a 2-branch spatial diversity can improve the link budget effectively, but it seems insufficient to secure a reliable wireless link for the capsule endoscope application. Nevertheless, it should be pointed out that if we employed higher order diversity or larger transmit power a higher date rate up to $10 \mathrm{Mbps}$ with longer link distance up to $15 \mathrm{~cm}$ could be expected.

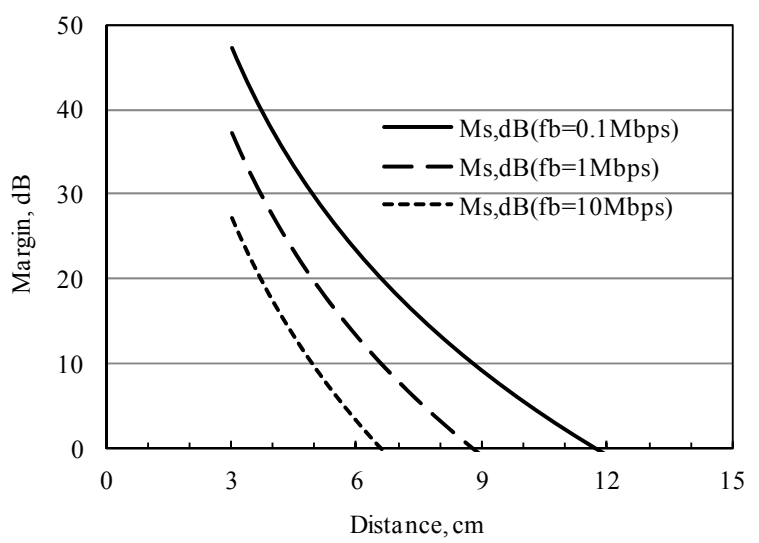

Figure 3. System margin versus communication distance for PPM-IR-UWB system

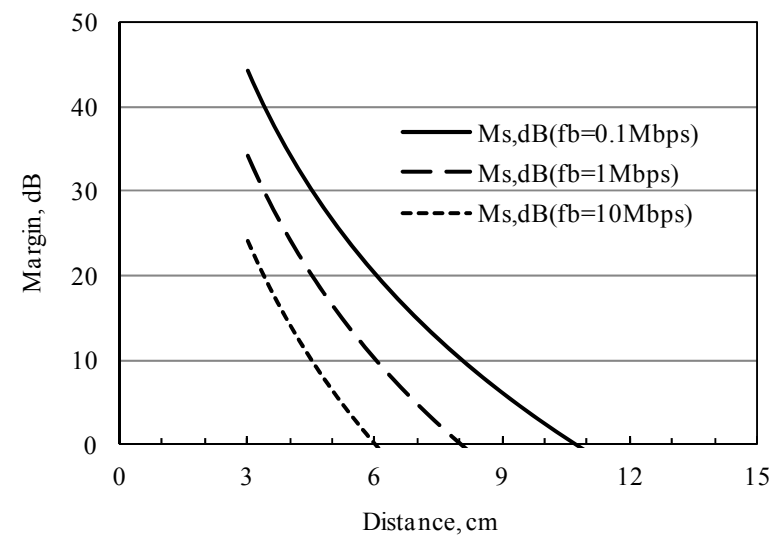

Figure 4. System margin versus communication distance for OOK-IR-UWB system

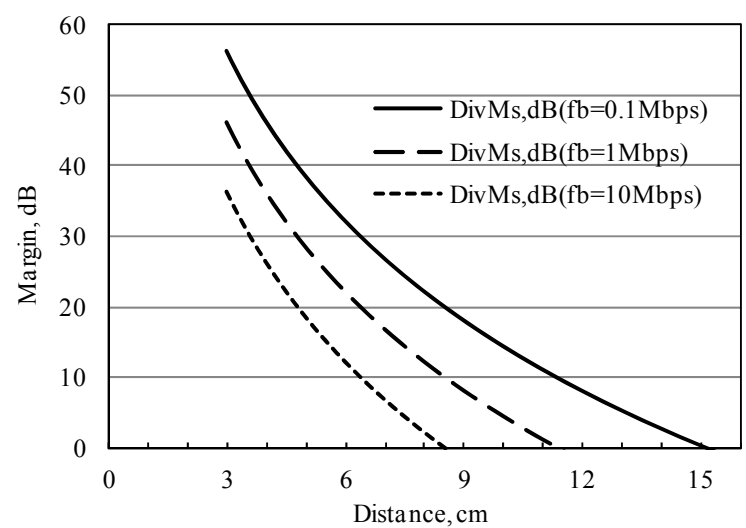

Figure 5. System margin versus communication distance for PPM-IR-UWB system with diversity 


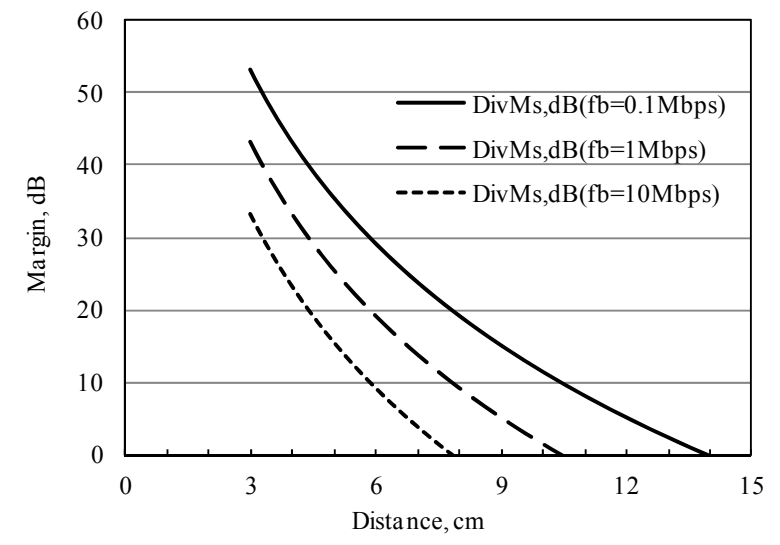

Figure 6. System margin versus communication distance for OOK-IR-UWB system with diversity

Table 2. Parameters for in-body to on-body UWB link budget analysis

\begin{tabular}{|c|c|c|c|c|}
\hline \multirow{2}{*}{ Data rate } & \multicolumn{4}{|c|}{ Maximum link distance [cm] } \\
\cline { 2 - 5 } & \multicolumn{2}{|c|}{ Without diversity } & \multicolumn{2}{c|}{ With diversity } \\
\cline { 2 - 5 } & PPM & OOK & PPM & OOK \\
\hline $0.1 \mathrm{Mbps}$ & 11.6 & 10.8 & 15.2 & 13.8 \\
\hline $1 \mathrm{Mbps}$ & 8.8 & 8 & 11.4 & 10.4 \\
\hline $10 \mathrm{Mbps}$ & 6.6 & 6 & 8.6 & 7.8 \\
\hline
\end{tabular}

\section{REQUIRED TRANSIT POWER AND DATA RATE}

The analysis in the previous section is under the condition that the allowable maximum transmitting power is $-10 \mathrm{dBm}$ based on the FCC UWB regulation. However, this regulation is defined in indoor environment. For a UWB transmitter inside the human body, the signals arriving to the body surface will be largely attenuated due to the lossy tissues. This means that a larger transmit power may be allowed as long as the signals at the body surface is sufficient weak. It is therefore meaningful to clarify the relationship between the data rate and the required transmit power. The required transmit power can be derived from Equation (3) and (4) as

$P_{t, d B W}=\left[E_{b} / N_{0}\right]_{d B}+N_{0, d B}+10 \log _{10} f_{b}+P L_{0, d B}+10 n \log _{10}\left(d / d_{0}\right)$

Under the 2-branch diversity reception of Rx1\&Rx3, we use Equation (6) to derive the relationship between required transmit power and data rate $f_{b}$ as an example. Here $E_{b} / N_{0}$ was set to $\left[E_{b} / N_{0}\right]_{\text {spec }}$ for obtaining a BER of 0.001 , i.e., $21 \mathrm{~dB}$ for IRUWB with PPM and $24 \mathrm{~dB}$ for IR-UWB with OOK. Figure 7 shows the required transmitting power as a function of date rate at a communication distance of $15 \mathrm{~cm}$, which covers almost all of the possible link distance for a capsule endoscope. The result indicates a required transmit power of at least $10 \mathrm{dBm}(10 \mathrm{~mW})$ for achieving a data rate as high as $10 \mathrm{Mbps}$ for the in-body to onbody transmission. Such a date rate can be expected to provide a high-quality image transmission for capsule endoscope application. Moreover, if the transmit power is allowed up to 20
$\mathrm{dBm}$, the data rate may achieve $50 \mathrm{Mbps}$ so that a real-time transmission for un-compressed capsule images become possible. It should be noted that, even if the transmit power $(20 \mathrm{dBm}$ or 100 $\mathrm{mW}$ ) is totally absorbed by the human body, the ten-gram averaged specific absorption rate (SAR) will be $10 \mathrm{~W} / \mathrm{kg}$ which still satisfies the safety guideline under occupational exposure condition. When the transmit power is limited below $10 \mathrm{dBm}$, the ten-gram averaged SAR will never exceed the safety guideline of $2 \mathrm{~W} / \mathrm{kg}$ for daily public exposure [10].

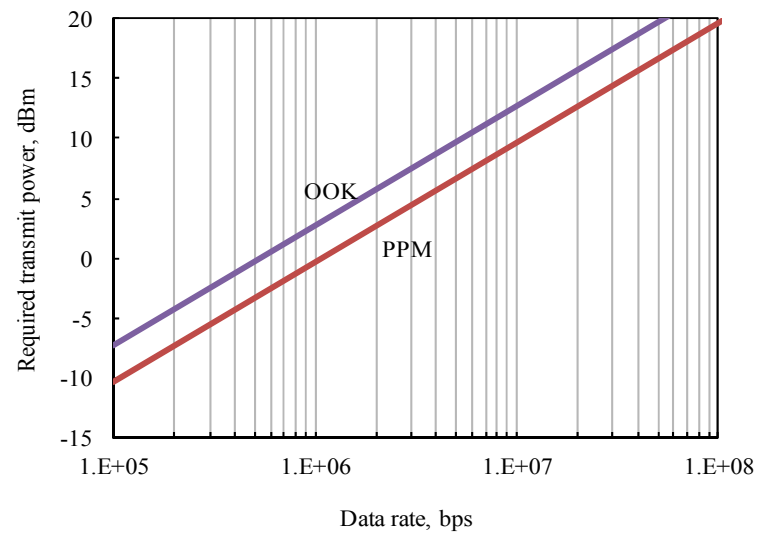

Figure 7. Required transmit power versus data rate at in-body to on-body communication distance of $15 \mathrm{~cm}$ for MRC diversity reception.

\section{CONCLUSIONS}

The link budget analysis for a typical medical application of capsule endoscope in UWB low band has been performed based on derived average BER performance of PPM and OOK schemes and non-coherent detection. Based on a predetermined system margin, the maximum link distance has been clarified at different data rates under the maximum allowable transmit power. In addition, the improvement on link distance by diversity reception has also been quantified, and the result has shown a sufficient feasibility to achieve a high data rate transmission at a desired link distance of $15 \mathrm{~cm}$. It has been found that if the transmit power is allowed up to $10 \mathrm{dBm}$, the data rate may achieve as high as $10 \mathrm{Mbps}$, and the SAR is still at a completely acceptable level.

\section{REFERENCES}

[1] A.W. Astrin, H.-B. Li and R. Kohno, "Standardization for body area networks," IEICE Trans. Commun., vol. E92-B, no. 2, pp.366-372, Feb. 2009.

[2] R. Chavez-Santiago, A. Khaleghi, I. Balasingham and T. A. Ramstad, "Architecture of an ultra wideband wireless body area network for medical applications," Presented at 2nd Int. Symp. on Applied Sciences in Biomedical and Communication Tech., Nov. 24-27, 2009, Bratislava, Slovak.

[3] J. Wang and Q. Wang, "Channel modeling and BER performance of an implant UWB body area link," Presented at 2nd Int. Symp. on Applied Sciences in Biomedical and Communication Tech., Nov. 24-27, 2009, Bratislava, Slovak.

[4] A. Khaleghi, R. Chavez-Santiago, X. Liang, I. Balasingham, V.C.M. Leung and T. Ramstad, "On ultra wideband channel modeling for in-body communications," Proc. 5th IEEE Int. 
Symp. on Wireless Pervasive Computing, May 5-7, 2010, Modena, Italy, pp.140-145.

[5] J. Shi, D. Anzai and J. Wang, "Diversity performance of UWB low band communication over in-body to on-body propagation channel," Presented at 6th EuCAP on Future Radios for Medical and Daily Healthcare Applications, Mar. 26-30, 2012, Prague, Czech Republic.

[6] T. Nagaoka, S. Watanabe, K. Saurai, E. Kunieda, S. Watanabe, M. Taki and Y. Yamanaka, "Development of realistic high-resolution whole-body voxel models of Japanese adult males and females of average height and weight, and application of models to radio-frequency electromagnetic-field dosimetry," Phys. Med. Biol., vol. 49, pp.1-15, 2004.

[7] J. Shi and J. Wang, "Channel characterization and diversity feasibility for in-body to on-body communication using low- band UWB signals", Presented at 3nd Int. Symp. on Applied Sciences in Biomedical and Communication Tech., Nov. 810, 2010, Roma, Italy.

[8] A. Sani, A. Alomainy, Y. Hao, "Numerical characterization and link budget evaluation of wireless implants considering different digital human phantoms," IEEE Trans. on Microwave Theory and Techniques, vol. 57, no. 10, Oct. 2009, pp. 2605-2612.

[9] Bic J.C., Duponteil D. and Imbeaux J.C. (1991): Elements of Digital Communication, John Wiley \& Sons Ltd., England, pp.462-467.

[10] ICNIRP 1998 Guidelines for limiting exposure to timevarying electric, magnetic, and electromagnetic fields (up to 300 GHz) Health Phys. 74 494-522. 